\title{
Simultaneous Inertia Force/Moment Balancing and Torque Compensation of Slider-Crank Mechanisms
}

\author{
Vigen Arakelian ${ }^{1}$ \\ Sébastien Briot ${ }^{2}$ \\ ${ }^{1}$ Département de Génie Mécanique et Automatique \\ Institut National des Sciences Appliquées (I.N.S.A.) \\ 20 avenue des buttes de Coësmes - CS 14315 \\ F-35043 Rennes, France \\ vigen.arakelyan@insa-rennes.fr \\ ${ }^{2}$ Institut de Recherches en Communications et Cybernétique \\ de Nantes (IRCCyN), UMR CNRS 6597 \\ 1 rue de la Noë, BP 92101, 44321 Nantes Cedex 3 France \\ sebastien.briot@irrcyn.ec-nantes.fr
}

Keywords: Slider-crank mechanism, shaking force balancing, shaking moment balancing torque compensation.

\begin{abstract}
This paper proposes a design concept which allows the simultaneous shaking force/shaking moment balancing and torque compensation in slider-crank mechanisms. At first, the shaking force and shaking moment are cancelled via a cam mechanism carrying a counterweight. Then, the spring designed for maintaining contact in this balancing cam mechanism is used for torque minimization. For this purpose, the spring is jointed with a second cam mounted on the input crank. The proposed design concept allows the development of only one device for solving the both mentioned problems. The suggested solution is illustrated by numerical example carried out by using ADAMS software.

\section{Introduction}

The slider-crank mechanisms are common elements in high-speed machines and many methods (Arakelian et al. 2000; Arakelian and Smith 2005; Lowen et al. 1983) have been developed for their balancing. The known methods can be arranged into the following groups:

a)Balancing by counterweights mounted on the links (Artobolevskii 1968; Campbell 1979; Berkof 1979a; Gheronimus 1968). The balancing methods based on the redistribution of mass of the mechanism by adding counterweights to links are well known. However, in the case of complete shaking force balancing, this approach is generally limited to simple mechanisms having only revolute joints. It is difficult to apply to mechanisms with a slider because the conditions for complete shaking force balancing bring about serious increase in the total mass of the balanced mechanism.

b) Harmonic balancing by counter-rotating masses (Artobolevskii 1968; Lanchester 1914; Shchepetilnikov 1982). These solutions are based on harmonic analysis. The reduction of inertia effects is primarily accomplished by the balancing of certain harmonics of the shaking forces and shaking moments. Unbalanced forces and moments are approximated by Fourier series (or Gaussian least-square formulation) and then each frequency component is studied. This solution has found wide application as it may be accomplished by attaching balancing elements to the crank. This approach has been used successfully for engine balancing. However, it is not applied on the off-set slider-crank mechanisms.

c)Self-balancing via a double mechanism (Artobolevskii 1968; Arakelian 1998; Arakelian 2006; Davies 1968; Dresig and Holzweißig 2004; Filonov and Petrikovetz 1987; Koropetz 1979; Turbin et al. 1978). The addition of an axially symmetric duplicate mechanism to any given mechanism will make the new combined center of mass stationary and thus balances the shaking force. This approach involves building self-balanced mechanical systems, in which two identical mechanisms execute
\end{abstract}


similar but opposite movements. In this case the shaking force is cancelled together with the shaking moment. A partial balancing is also possible by this approach.

d) Balancing by added dyad (Arakelian 1998; Arakelian and Smith 1999; Doronin and Pospelov 1991; Frolov 1987). The parallelogram loop, consisting of the initial links of the mechanism and the added dyad, transfers the motion of the coupler link to a shaft on the frame, where it is connected to a counterweight of considerably reduced mass. Partial balancing may be achieved by the generation of an approximate straight-line movement of a counterweight mounted on the added dyad. Among several works may be distinguished also the studies in which pantograph mechanism properties are used. The aim of this approach is to balance the mechanism by using the copying properties of the pantograph formed from the links of the initial mechanism and added links. The pantograph carries a counterweight that achieves the condition necessary for shaking force and shaking moment balancing.

e)Balancing by planetary systems (Arakelian and Smith 1999; Berestov 1978; Gao 1990; Ye and Smith 1994). The application of planetary systems allows the cancellation of the shaking moment of mechanisms. However, such a balancing can only be reached by a considerably complicated design of the initial mechanism.

f) Balancing by using a cable and pulley arrangement (Berkof 1979b). In this case the opposite motions of the balancing counterweight and the slider is achieved via a cable and pulley arrangement.

g) Balancing by using a cam mechanism (Kamenski 1968; Kato 1995; Kato 1997; Krause 1987; Schrick and Hanula 1995). In this approach the reduction of inertia forces has been performed by means of a cam carrying a counterweight and it was shown how cam-driven masses may be used to keep the total center of mass of a mechanism stationary.

It is known that the inertia force balancing can be only achieved by adding complementary masses and it brings an increase in the input torque. The input torque may be reduced by optimal redistribution of moving masses (Arakelian 2007; Berkof 1979b; Chaudhary 2007; Soong 2001; Yan and Soong 2001) or by using non-circular gears (Yao and Yan 2003). One of the more efficient methods used to solve the problem of input torque balancing is creating a cam-spring mechanism, in which the spring is used to absorb the energy from the system when the torque is low, and release energy to the system when the required torque is high. It allows reducing the fluctuation of the periodic torque in the high-speed mechanical systems (Angeles and Wu 2001; Arakawa et al. 1997; Benedict et al. 1971; Benedict and Tesar 1970; Funk and Han 1996; Guilan et al. 1999; Nishioka 1999; Nishioka and Yoshizawa 1995; Poludov 1979).

In mechanical design, these two problems are considered separately, i.e. the mechanism can be balanced by mentioned methods and, after, its input torque can be compensated by a cam-spring mechanism.

In this paper, a new design approach is developed, which proposes simultaneous inertia force balancing and torque compensation in slider-crank mechanisms.

\section{Design of the inertia force/moment balanced and torque compensated slider-crank mechanism}

Fig. 1 shows an off-set slider crank mechanism, which contains an initial slider-mechanism $O A B$ with crank 1 mounted on the frame, rod 2 and slider 3 , as well as cams 4,5 with followers 6,7 and a compression spring 8 .

Let us first consider the inertia force and moment balancing of the slider-crank mechanism. For this purpose, we consider that rod 2 is a "physical pendulum" link (Berkof 1973) (see also (Arakelian 2007)), i.e. its mass distribution allows the dynamic substitution of the rod's mass by two point masses, also,

$$
\left[\begin{array}{ccc}
1 & 1 & 1 \\
l_{A S 2} & 0 & -l_{B S 2} \\
l_{A S 2}^{2} & 0 & l_{B S 2}^{2}
\end{array}\right]\left[\begin{array}{c}
m_{A} \\
0 \\
m_{B}
\end{array}\right]=\left[\begin{array}{c}
m_{2} \\
0 \\
I_{S 2}
\end{array}\right]
$$

where, $m_{A}$ and $m_{B}$ are point masses; $m_{2}$ is the mass of rod $2 ; I_{S 2}$ is the axial moment inertia of the rod about the centre of mass $S_{2}$ of the link; $l_{A S 2}$ and $l_{B S 2}$ are the distances between the centres of the joints $A$ and $B$ and the centre of mass $S_{2}$ of link 2, respectively. 


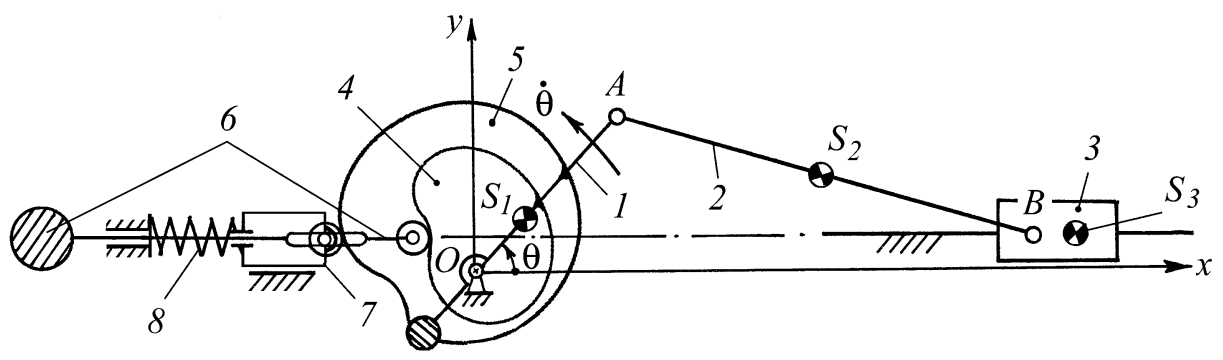

Fig. 1. Balanced and torque compensated off-set slider-crank mechanism.

Thus, the dynamic model of the rod represents a weightless link with two point masses $m_{A}$ and $m_{B}$ situated in the centres of corresponding joints. This dynamic model is fully equivalent to the real rod.

We now require rotating masses to be balanced about point $O$. Therefore, the displacement of the counterweight mounted on follower 6 is selected in such a manner that the inertia force of the follower 6 with counterweight will be opposite to the inertia force of the masses $m_{3}$ and $m_{B}$ carried out reciprocating motion:

$$
\ddot{x}_{S 6}=-\frac{m_{B}+m_{3}}{m_{6}} \ddot{x}_{B} .
$$

where $m_{3}$ is the mass of the slider $3, m_{6}$ the mass of follower 6 with counterweight, $\ddot{x}_{s 6}$ the acceleration of the follower 6 and $\ddot{x}_{B}$ the acceleration of the slider.

Therefore, in order to generate a prescribed reciprocating motion of follower 6 with acceleration $\ddot{x}_{s 6}$, the cam 4 is used. Then, by means of a counterweight, the centre of mass of crank 1 plus the cam 4 and point mass $m_{A}$ is brought to the centre of the pivot $O$.

After such a redistribution of moving masses, the shaking force and shaking moment are cancelled and the slider-crank mechanism transmits no inertia loads to surrounding. However, it is known that the added masses destined to balance the inertia force increase the input torque of the mechanism. For minimisation of the input torque of mechanism, one uses another technique which consists in adding a cam-spring compensation device.

The above-mentioned literature review shows that these two problems, i.e. inertia force balancing and torque compensations, are studied separately and it is considered that they are not coupled. Thus, according with the known design approaches, two devices will be developed and coupled with the slider-crank mechanism.

It will be show that the design of these two problems can be considered together and the spring used for maintaining the contact between the counterweight and the cam may also be used for balancing the input torque. For this purpose, we use the elastic force of spring 8 designed for maintaining contact between follower 6 and cam 4 . In other words, we will use the spring 8 for the generation of a complementary moment on the input crank.

Let us now consider the input torque compensation.

The input torque $\tau$ of the dynamically balanced mechanism with the spring can be written under the form:

$$
\tau=\frac{\mathrm{d}}{\mathrm{d} t}\left(\frac{\partial L}{\partial \dot{\theta}}\right)-\frac{\partial L}{\partial \theta}
$$

where $L=T-V$ is the Lagrangian of the system, $T$ is kinetic energy and $V$ its potential energy. Neglecting the mass of the spring and follower 7, $T$ and $V$ can be written as:

$$
\begin{gathered}
T=0.5\left(I_{S 1} \dot{\theta}^{2}+m_{1} \dot{x}_{S 1}^{2}+m_{c p} \dot{x}_{c p}^{2}\right)+0.5\left(I_{S 2} \dot{\theta}_{2}^{2}+m_{2} \dot{x}_{S 2}^{2}\right)+0.5 m_{3} \dot{x}_{B}^{2}+0.5 m_{6} \dot{x}_{S 6}^{2} \\
=0.5\left(I_{S 1}+\left(m_{c p} r_{c p}^{2}+m_{1} r_{1}^{2}+m_{A}\right) L_{O A}^{2}\right) \omega^{2}+0.5\left(m_{B}+m_{3}\right)\left(1+\frac{m_{B}+m_{3}}{m_{6}}\right) \dot{x}_{B}^{2} \\
V=0.5 k \delta^{2},
\end{gathered}
$$

where

$-\omega=\dot{\theta}$ is a constant for steady-state conditions, 
- $\dot{x}_{S 2}$ is the velocity of the centre of masses of element 2 and $\dot{\theta}_{2}$ its angular velocity

$-k$ is the spring constant,

- $\delta$ is the displacement of the end of the spring from its equilibrium position,

- $L_{O A}$ is the distance between joint centres $O$ and $A$,

- $m_{c p}$ is the mass of the counterweight mounted on the element 1 ,

$-r_{c p}$ is the dimensionless position of this counterweight $\left(r_{c p}=\left(m_{1} l_{O S 1} / l_{O A}+m_{A}\right) / m_{c p}\right)$,

- $m_{1}$ is the mass of the element 1 plus the cam 4 and $I_{S 1}$ their global axial moment of inertia,

- $r_{1}$ is the dimensionless position of the global mass centre of these elements $\left(r_{1}=l_{O S 1} / l_{O A}\right)$.

Thus, the first and second terms of the Lagrange equation (3) become:

$$
\begin{gathered}
\frac{\mathrm{d}}{\mathrm{d} t}\left(\frac{\partial L}{\partial \dot{\theta}}\right)=2\left(m_{B}+m_{3}\right)\left(1+\frac{m_{B}+m_{3}}{m_{6}}\right)\left(\frac{\partial x_{B}}{\partial \theta}\right)\left(\frac{\partial^{2} x_{B}}{\partial \theta^{2}}\right) \omega^{2} \\
\frac{\partial L}{\partial \theta}=\left(m_{B}+m_{3}\right)\left(1+\frac{m_{B}+m_{3}}{m_{6}}\right)\left(\frac{\partial x_{B}}{\partial \theta}\right)\left(\frac{\partial^{2} x_{B}}{\partial \theta^{2}}\right) \omega^{2}-k \frac{\partial \delta}{\partial \theta} \delta
\end{gathered}
$$

taking into account that

$$
x_{B}=L_{O A} \cos \theta \pm \sqrt{L_{A B}^{2}-\left(a-L_{O A} \sin \theta\right)^{2}}
$$

$L_{A B}$ being the distance between joint centres $A$ and $B$.

Therefore, the input torque can be deduced:

$$
\tau=\left(m_{B}+m_{3}\right)\left(1+\frac{m_{B}+m_{3}}{m_{6}}\right)\left(\frac{\partial x_{B}}{\partial \theta}\right)\left(\frac{\partial^{2} x_{B}}{\partial \theta^{2}}\right) \omega^{2}+k \frac{\partial \delta}{\partial \theta} \delta
$$

In order to avoid torque fluctuation, the spring has to create a force that makes the input torque constant. In many cases, when a mean value of the torque moment is equal to zero, as in the case of unloaded slider-crank mechanism, this constant is equal to zero. Thus, in such a case, the compensation of the input torque is equivalent to its cancellation. From the point of view of the energy fluctuation, after such a compensation, the periodic variations of the input torque is cancelled and the required input torque is equal to zero, i.e. in the stationary operating mode, the generation of motion may be accomplished by only a very small input torque, which is needed for overcoming friction.

Thus, under the condition that the input torque is equal to zero with the cam-follower system, equation (9) admits the integral:

$$
\left(m_{B}+m_{3}\right)\left(1+\frac{m_{B}+m_{3}}{m_{6}}\right)\left(\frac{\partial x_{B}}{\partial \theta}\right)^{2} \omega^{2}+k \delta^{2}=A,
$$

where $A$ is an integration constant. Note that the value of $A$ represents two times the sum of the kinetic energy stored in the mechanism (without rod 1 and the cam 5) plus the potential energy in the spring.

For the continuity of contact between the cam and the follower, the force created by the spring has to be always superior to the inertia force of the follower 6 with the counterweight, i.e.

$$
k \delta \geq\left(m_{3}+m_{B}\right) \ddot{x}_{B}
$$

Also,

$$
\delta^{2} \geq \frac{\left(m_{3}+m_{B}\right)^{2}}{k^{2}} \ddot{x}_{B}^{2}
$$

From expression (8), the value of $\delta$ may also be computed:

$$
\delta^{2}=\frac{A-\left(m_{B}+m_{3}\right)\left(1+\frac{m_{B}+m_{3}}{m_{6}}\right)\left(\frac{\partial x_{B}}{\partial \theta}\right)^{2} \omega^{2}}{k}
$$

Combining equations (12) and (13) leads to,

$$
A \geq\left(m_{B}+m_{3}\right)\left(\left(1+\frac{m_{B}+m_{3}}{m_{6}}\right)\left(\frac{\partial x_{B}}{\partial \theta}\right)^{2}+\frac{m_{3}+m_{B}}{k} \frac{\partial^{2} x_{B}}{\partial \theta^{2}}\right) \omega^{2}
$$

In order equation (17) to be valuable for any value of $\theta$, constant $A$ should be equal to: 


$$
A=\max _{\theta \in[0,2 \pi]}\left(\left(m_{B}+m_{3}\right)\left(\left(1+\frac{m_{B}+m_{3}}{m_{6}}\right)\left(\frac{\partial x_{B}}{\partial \theta}\right)^{2}+\frac{m_{3}+m_{B}}{k} \frac{\partial^{2} x_{B}}{\partial \theta^{2}}\right) \omega^{2}\right)
$$

To avoid resonance, the spring should be stiff enough so that the lowest natural frequency of the system is considerably higher than the highest significant harmonic of the output motion of the follower (Angeles and Wu 2001). After the appropriate spring constant is selected, the displacement of the spring can be determined from equations (13) and (15), namely:

$$
\delta=\sqrt{\frac{A-\left(m_{B}+m_{3}\right)\left(1+\frac{m_{B}+m_{3}}{m_{6}}\right)\left(\frac{\partial x_{B}}{\partial \theta}\right)^{2} \omega^{2}}{k} .}
$$

Thus here we find the cam profile for torque compensation.

The next part presents an illustrative example of the proposed approach. The simulations have been carried out by using ADAMS software.

\section{Illustrative example}

\subsection{Shaking force and shaking moment balancing}

The following parameters of mechanism's links are specified for the simulations: $L_{O A}=0.292 \mathrm{~m}$; $L_{A B}=0.427 \mathrm{~m} ; r_{1}=0.5 ; y_{B}=0.1 \mathrm{~m} ; m_{1}=2 \mathrm{~kg} ; m_{2}=3 \mathrm{~kg} ; m_{3}=4 \mathrm{~kg} ; I_{S 1}=0.03 \mathrm{~kg} / \mathrm{m}^{2}, I_{S 2}=0.14$ $\mathrm{kg} / \mathrm{m}^{2}$. The period of the mechanism is fixed to $1 \mathrm{~s}$.

The shaking forces and shaking moment of the above-mentioned mechanism are represented in Fig. 2 (full line).

By selecting $L_{c p}=r_{c p} L_{O A}=0.2 \mathrm{~m}$, we obtain $m_{c p}=3.25 \mathrm{~kg}$. This counterweight will be mounted on the input crank. The second counterweight is carried out the reciprocating motion. In order to reduce the size of the cam, the displacement of the centre of mass $S_{6}$ of link 6 is three times smaller than the displacement of point $B$. Therefore $m_{6}=13.5 \mathrm{~kg}$. Fig. 3 shows the obtained cam profile. The variations of the shaking forces and shaking moment of the mechanisms with redistributed moving masses are given in Fig. 2 by dashed lines. The simulation results show that after balancing the shaking forces and moment are cancelled.

\subsection{Input torque compensation}

The parameters are identical to those used in the previous part. The constant spring is fixed to $k=$ $10 \mathrm{kN} / \mathrm{m}$. The joint between the cam and the follower has been modeled by a contact between two solid bodies. The linear spring 8 is designed in such a manner that it ensures a permanent contact in the cam mechanisms. Thus, the displacements of the follower 7 are determined from equation (16) and the obtained cam profile is shown in Fig. 4.

The input torques before and after compensation are shown in Fig. 5.

Thus we can note that the suggested approach allows not only to carry out complete shaking force and shaking moment balancing of the of-set slider crank mechanism but also it assumes its input torque compensation.

\section{Conclusions}

Fast-moving machinery with rotating and reciprocating masses is a significant source of variable dynamic loads. A major theme in machine dynamics and machine design is seeking to minimize the fluctuating forces that such machinery applies to its environment via its mount. Another theme, which is also very important in machine dynamics, is the minimization of the input torque fluctuation caused by the variable dynamic loads. These two problems are known and many methods have been developed and documented. However, these themes are considered separately, as two decoupled problems. 
In this paper, for the first time, simultaneous shaking force/shaking moment balancing and torque compensation in slider-crank mechanisms is considered. The shaking force and shaking moment are cancelled via a cam mechanism carrying a counterweight. Then, the spring designed for maintaining contact in this balancing cam mechanism is used for torque compensation. For this purpose, the spring is jointed with a second cam mounted on the input crank. The proposed design concept allows the development only one devise for solution of the both mentioned problems.

The suggested solution is illustrated by simulations carried out for an off-set slider-crank mechanism.

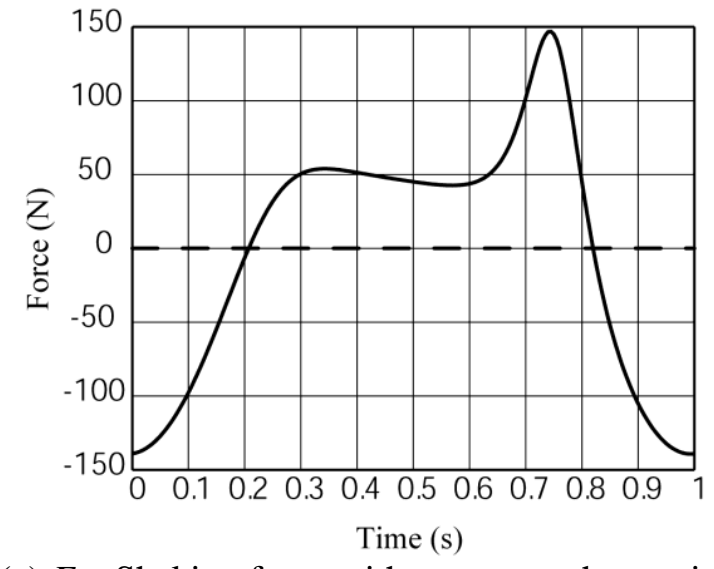

(a) $F_{x}$ : Shaking force with respect to the $x$ axis.

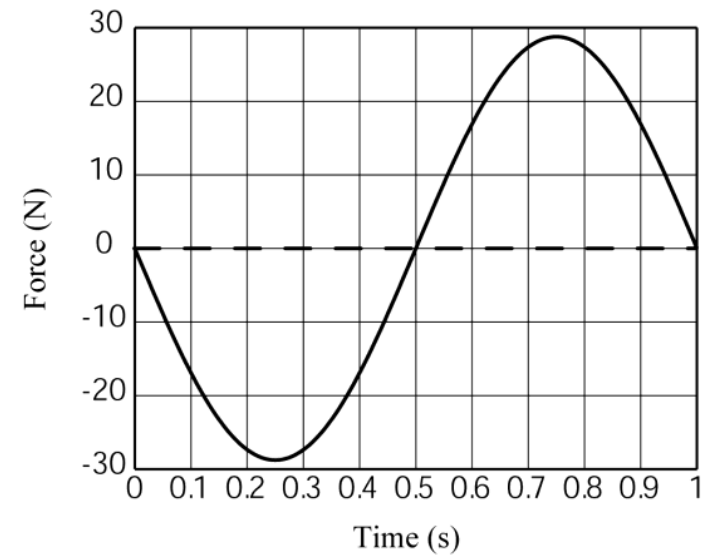

(b) $F_{y}$ : Shaking force with respect to the $y$ axis.

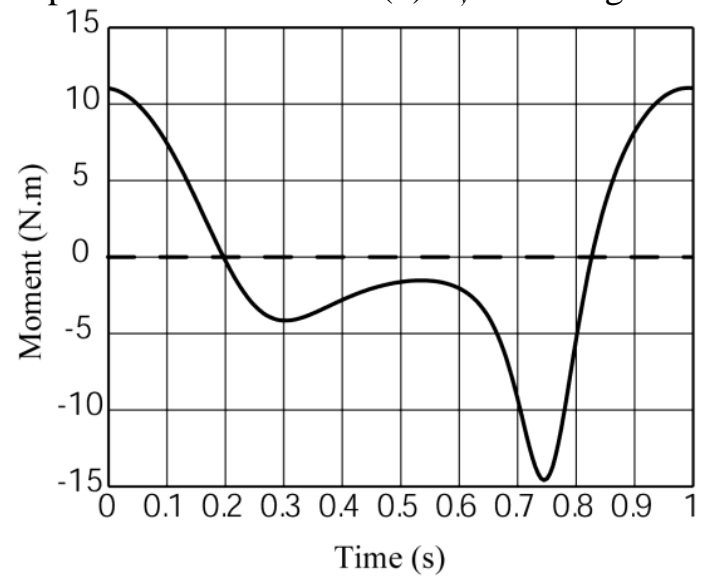

(c) $M$ : Shaking moment

Fig. 2. Variations of the shaking forces and shaking moment before (full line) and after (dashed line) balancing.

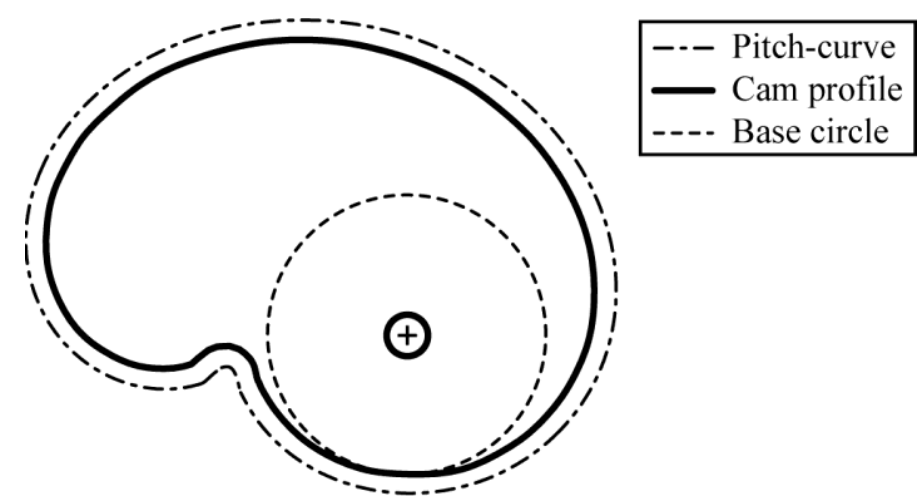

Fig. 3. Profile of the cam for the displacement of link 6 assuming the inertia forces balancing. 


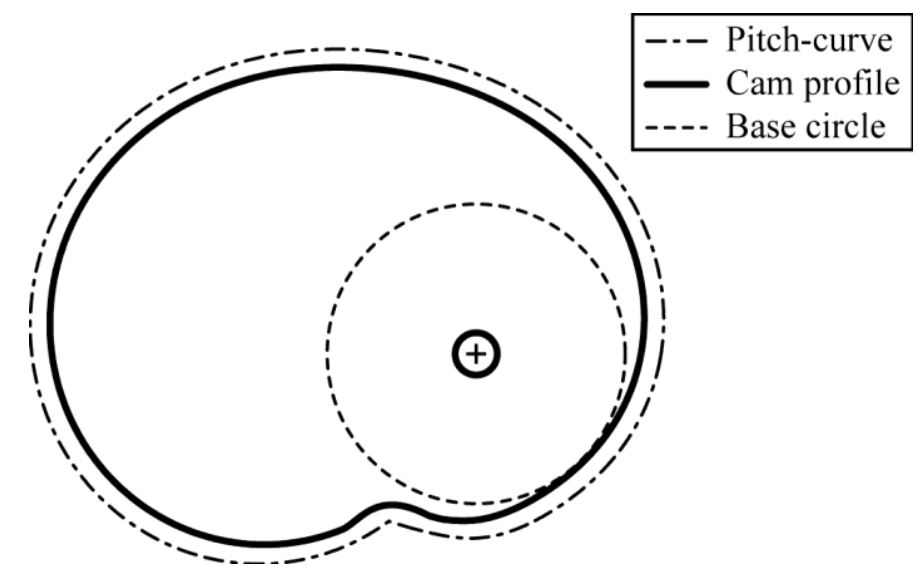

Fig. 4. Profile of the cam for the displacement of link 7 assuming the torque compensation.

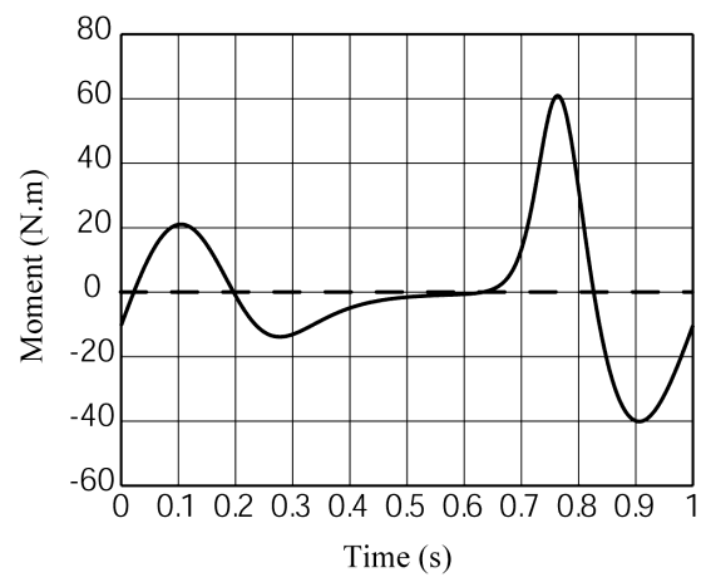

Fig. 5. Input torque before (full line) and after (dashed line) compensation.

\section{References}

Arakawa, M., Nishioka, M., Morita, N., 1997. Torque compensation cam mechanism. Proc. Joint International Conf. on Advanced Science and Technology, Zhejiang University, Hangzhou, China, 302-305.

Angeles, J., Wu, C.-J., 2001. The optimum synthesis of en elastic torque-compensating cam mechanism. Mechanism and Machine Theory 36, 245-259.

Arakelian, V., 1998. Equilibrage dynamique complet des mécanismes. Mech. and Mach. Theory 33 (4), 425-436.

Arakelian, V. 2006. Shaking moment cancellation of self-balanced slider-crank mechanical systems by means of optimum mass redistribution. Journal of Mechanics Research Communications 33, 846850.

Arakelian, V., 2007. Complete shaking force and shaking moment balancing of RSS'R spatial linkages. Multi-body Dynamics Part K 221, 303-310.

Arakelian, V., Dahan, M., Smith, M.R., 2000. A historical review of the evolution of the theory on balancing of mechanisms. International Symposium on History of Machines and Mechanisms Proceedings HMM2000, Kluwer Academic Publishers, Dordrecht / Boston / London, 291-300.

Arakelian, V., Smith, M., 1999. Complete shaking force and shaking moment balancing of linkages. Mechanism and Machine Theory 34 (8), 1141-1153. 
Arakelian, V., Smith, M.R., 2005. Shaking force and shaking moment balancing of mechanisms: an historical review with new examples. Transactions of the ASME. Journal of Mechanical Design 127, 334-339.

Artobolevskii I.I., 1968. Theory of mechanisms and machines. Moscow, Ed. Nauka, 644p.

Berestov, L. V., 1978. Shaking force and shaking moment balancing in planar mechanisms. Ph.D. Thesis, State University of Kazakhstan, Alma-Ata, 203p.

Benedict, C.E., Matthew, G.K., Tesar, D., 1971. Torque balancing of machines by sub-unit cam systems. 2nd Applied Mechanism Conference, paper No. 15, Oklahoma State University, Stillwater, Oklahoma.

Benedict, C.E., Tesar, D., 1970. Optimal torque balance for a complex stamping and indexing machine. Mechanisms Conference, Paper No. 70-Mech-82, Columbus, Ohio.

Berkof, R. S., 1973. Complete force and moment balancing of inline four-bar linkages. Mechanism and Machine Theory 8, 397-410.

Berkof, R.S., 1979a. Force balancing of a six-bar linkage. Proceedings of the Fifth World Congress on Theory of Machines and Mechanisms, 1082-1085.

Berkof, R.S., 1979b. The input torque in linkages. Mechanism and Machine Theory 14, 61-73.

Campbell, D.N., 1979. Balanced slider-crank mechanism. Patent FR 2421301.

Chaudhary, H., 2007. Balancing of four-bar linkages using maximum recursive dynamic algorithm. Mechanism and Machine Theory 42, 216-232.

Davies, T.H., 1968. The kinematics and design of linkages, balancing mechanisms and machines. Machine Design Eng., 40, 40-51.

Doronin, V.I., Pospelov, A.I., 1991. Balanced slider-crank mechanism. Patent SU1627769.

Dresig, H. and Holzweißig, F. 2004. Maschinendynamik. Springer, 526p.

Filonov, I.P., Petrikovetz, I.P., 1987. Balancing device of lever mechanisms. Patent SU1296762.

Frolov, K.V., 1987. Theory of mechanisms and machines. Moscow, Ed. "Vishaya shkola", 496p.

Funk, W., Han, J., 1996 On the complete balancing of the inertia-caused input torque for plane mechanisms. Proceedings of the Design Engineering Technical conference, Irvine, California.

Gao, F., 1990. Complete shaking force and shaking moment balancing of 26 types of four-, fiveand six-bar linkages with prismatic pairs. Mechanism and Machine Theory 23 (2), 183-192.

Gheronimus Y.L., 1968. An approximate method of calculating a counterweight for the balancing of vertical inertia forces. Mechanisms 3 (4), 283-288.

Guilan, T., Haibo, F., Weiyi, Z., 1999. A new method of torque compensation for high speed indexing cam mechanisms, ASME Journal of Mechanical Design 121, 319-323.

Kamenski, V.A., 1968. On the question of the balancing of plane linkages. Mechanisms 3 (4), 303 322.

Kato H., 1995. Mechanical press. Patent DE4430244.

Kato H., 1997. Mechanical pressing machine with dynamic balancing device. Patent US5605096.

Koropetz, A.A, 1979. Shaking force and shaking moment balancing of the mechanisms of machines for the scouring grain. Moscow, Ed. NTVIM 13, 62-71.

Krause, H.H., 1987. Device for balancing inertia forces and mass moments of inertia, Patent DE3607133.

Lanchester, F.M., 1914. Engine balancing. Horseless Age, 33 (12-16), March 25, April 1, 8, 15, 22, 494-498, 536-538, 571-572, 608-610, 644-646.

Lowen, G.G., Tepper., F.R., Berkof, R.S., 1983. Balancing of linkages - an update. Mech. and Mach. Theory 18 (3), 213-230.

Nishioka, M., 1999. Design of torque compensation cam using measured torque distribution. Proceedings of the 10th World Congress on the Theory of Machines and Mechanisms, 20-24 June, Oulu, Finland, pp.1471-1476.

Nishioka, M., Yoshizawa, M., 1995. Direct torque compensation cam mechanisms. Transactions of the Japan Society of Mechanical Engineers 61 (585), 2020-2024.

Poludov, A.N., 1979. Systèmes de décharge programmables des mécanismes cycliques. Ed.: "Bischa Chkola", Lvov.

Schrick, P., Hanula B., 1995. Free inertia forces balancing piston engine. Patent WO9526474.

Shchepetilnikov, V.A., 1982. Balancing of mechanisms. Moscow, Ed. Mashinostroenie, 256p. 
Soong, R.C., 2001. Minimization of the driving torque of full force balanced four-bar linkages. Journal of Kao Yuan Institute of Technology, 591-594.

Turbin, B.I, Koropetz, A.A., Koropetz, Z.A., 1978. The possibility of the shaking force balancing in the system with oscillating links. Russian Journal Mechanism and Machine Theory 7, Moscow, 87-90.

Yan, H.S., and Soong, R.C., 2001. Kinematic and dynamic design of four-bar linkages by links counterweighing with variable input speed. Mechanism and Machine Theory 36 (9), 1051-1071.

Yao, Y.A., Yan, H.S., 2003. A new method for torque balancing of planar linkages using noncircular gears. Journal of Mechanical Engineering Science Part C 217 (5), 495-503.

Ye, Z., Smith, M., 1994. Complete balancing of planar linkages by an equivalent method. Mechanism and Machine Theory 29 (5), 701-712. 